\title{
Mobile and Web Architectures of Vehicle Tracking and Human-Object Tracking Systems in Wireless Sensor Networks
}

\author{
Murat Dener
}

\begin{abstract}
With the recently increasing theft and kidnapping incidents the use of tracking systems has begun to increase. In line with this demand the tracking systems have also been rapidly improved and new systems have been produced. Two of the most widely used tracking systems are vehicle tracking and human-object tracking systems. Thanks to these systems one can either track his/her own vehicle or the members of the family, and if desired, fleet of vehicles or cargo tracking can also be made. Vehicle and human-object racking systems can position locations with the help of wireless network sensors and GPS modules on them and these location can be displayed/tracked on the web or mobile platforms generated. In this study displaying of vehicle and human-object tracking systems created with wireless sensor networks on web or mobile platforms has been implemented.
\end{abstract}

Index Terms-Human and object tracking systems, map, vehicle tracking on web or mobile devices, wireless sensor networks.

\section{INTRODUCTION}

Advanced Technologies make our lives easy day by day. One of the technologies that make our lives easy is wireless sensor networks. With the use of wireless Sensor Networks GPS data can be retrieved from distant vehicles, humans or objects and these data, if desired, can be displayed on web or mobile platforms. Wireless Sensor Networks are formed by means of connecting many sensor nodes in a wireless connection. These nodes can make data communications possible from one region to the other over a network they form in a wide area. In this study, the process of displaying the data taken from the GPS module has been implemented on web and mobile platforms. Android operating system has been used as the mobile operating system. As per the report of IDC [1], the Android operating system dominates $84 \%$ of the market. For this reason, the Android operating system is the one with the largest number of users. Android is a mobile operating system with open source coded made using Linux 2.6 kernel [2]. The data collected from wireless sensor networks are sent to the database over GSM/GPRS/GPS modules. The Android and web application written extracts the data sent from the database using web services. The extracted data are displayed on a map of the Android and web interface. In line with the study conducted, instant readings can be displayed through the Android and web interfaces

Manuscript received February 5, 2016; revised September 16, 2016.

Murat Dener is with the Graduate School of Natural and Applied Sciences, Gazi University, Ankara, Turkey (e-mail muratdener@gazi.edu.tr). using sensor network infrastructure which receives information about the position or speed of a vehicle fleet. In the second part of the study, some information has been given about wireless sensor networks. In part three, information has been given about Vehicle Tracking Systems and working principles of a vehicle tracking systems has also been mentioned. In part four however, human and Object tracking systems have been mentioned and information on working principles has been given. Conclusions and recommendations have been presented on the last part.

\section{WIRELESS SENSOR NETWORKS}

Wireless Sensor Networks are data transmission tools which provide wireless data communication and over which many nodes communicate [3]. By adding many sensors on wireless sensor networks different data communications can be implemented. For instance; many sensors such as temperature, light, pressure, heat and gas sensors can be interactively used with wireless sensor networks. Apart from these sensors, the wireless sensor networks can also work interactively with GPS modules. Information on the position coordinates of a node can be instantly sent through the GPS module positioned on a wireless sensor network. Thanks to this technology, tracking of vehicles, humans and any object desired can be made. The sensor node, by virtue of the GPS module on it, relays the coordinate data firstly to the database server, that way the coordinate data can be pulled from the database and can be displayed on a map through web as well as mobile platforms (Fig. 1).

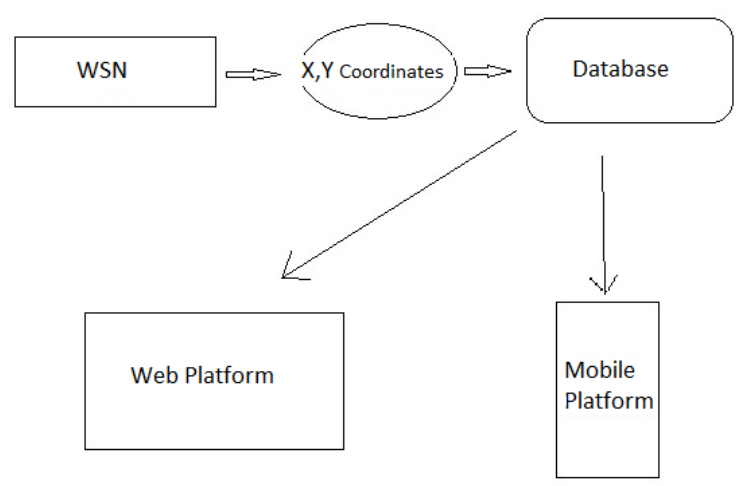

Fig. 1. GPS data communication and working principle.

Location data are extracted instantly from the database and displayed as an icon on a map on the mobile and android applications. The icon of the vehicle or the object moves continuously depending on the location data received. 


\section{ZIGBEE}

Data communication is wirelessly carried out with Zigbee technology. Givenbelow is the information that is needed to be known about ZigBee. The ZigBee;

- Is a wireless communication protocol standardised by IEEE on the code 802.15.4.

- Works at three different speeds in $868 \mathrm{MHz}-20 \mathrm{~kb} / \mathrm{s}, 915$ $\mathrm{MHz}-40 \mathrm{~kb} / \mathrm{s}$ and $2.4 \mathrm{GHz}-250 \mathrm{~kb} / \mathrm{s}$.

- Supports 1- 75 metre distances.

- Has a battery life of 6 months to 2 years.

- Uses a strong encrypting method (AES-128).

- Is low cost.

- Is easy to install.

The IEEE 802.15.4 [4,5] standard has a multiple network topology. These network topologies include star connection and point-to-point connection schemes. When the advantages such as reliability, low cost and energy savings are taken into account, the Zigbee can be used for the wireless connections of the sensors and management devices just like PC input devices. Zigbee enables the automatically search of the wireless communication channels and existence of many wireless networks concurrently. The Zigbee products use the $2.4 \mathrm{GHz}$ frequency band which is open for use worldwide. In addition to this, $915 \mathrm{MHz}$ can be used in American Continents and 868 in Europe. It can reach up $250 \mathrm{Kbps}$ on $2.4 \mathrm{GHz}$ frequency with ten channels, $40 \mathrm{Kbps}$ on $915 \mathrm{GHz}$ frequency with six channels and $20 \mathrm{Kbps}$ on $868 \mathrm{GHZ}$ frequency with one channel. The access distance of the products varies between 10 to 75 metres depending on the transmission power and ambient conditions. Depending on the flow of the files, the Zigbee devices provide power savings by hibernating. By this means, the battery life can reach up to months (Even up to a year) with the hibernation periods lasting for hours and with an ideal connection technique.

The aim of Zigbee is to offer a network protocol to devices which are not used frequently but need to be remote controlled or to point sensors. The most well-known rival of it is actually Bluetooth, but as they don't cater for the same market, it is only in 1/6 of the speed and the coverage area is wider than Bluetooth. The difference however, is that it consumes incredibly less power and the fact that it will most likely be used with non-chargeable batteries. The area of use of Zigbee is so wide-ranged, these could be home devices to some parts of cars. Consequently, every zone that you want information to come from is suitable for Zigbee. Also as it is wireless and cheap, it can also decrease the cabling costs. As a matter of fact, some models can also be designed to produce its own power.

\section{GPS}

Global Positioning System is a satellite network that sends encoded information regulary. It measures to distance between the satellites and the GPS sensor (signal strength). The system consists of 24 satellites. GPS sensors calculates to location determination process with an algorithm according to the signal of the satellite. When the GPS sensor receives signals from at least four different satellites can calculate its location on Earth. In case of connecting more satellites to calculate the position with less error. The accuracy of GPS data ranges from 1 to $100 \mathrm{~m}$. This error value may vary depending on conditions such as the speed of the sensor. environmental conditions, the number of satellites (Fig. 2).

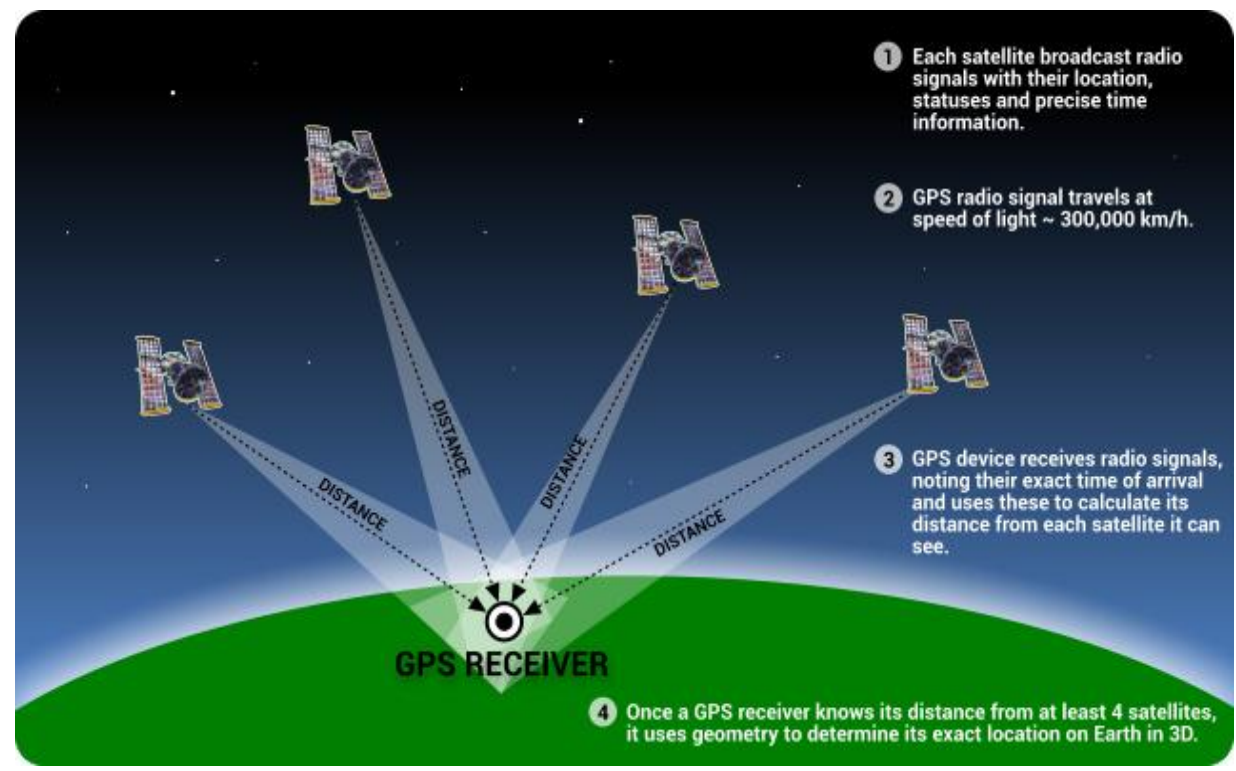

Fig. 2. GPS receiver.

NMEA is called the data sent by GPS. There are 19 different NMEA standard. All GPS sensors working on this standard, this standard GPS output data are sent.

\section{Vehicle Tracking System}

It is now possible to determine the position of all vehicles by virtue of Vehicle Tracking Systems. By means of the GPS position data coming to the system the coordinates can be displayed as well as the position can be determined on the map. For a vehicle tracking system, wireless sensor networks can be used and this is preferable due to its features such as its low cost, easy installation and flexibility. By the virtue of the GPS component/module installed on the wireless sensor 
nodes, the position data can be sent. This position data is possible to be displayed on a map. Most of the vehicle tracking systems available on the market use different map applications. Most widely used of these is "Google Maps API" of Google. With the making of some minor changes on the map, whether the position is determined and displayed on the map or the tracking can be made by the continuously updated position data or the features of the vehicle being tracked, such as the speed, the direction headed too, can be seen on the map. In Fig. 3, is the web application of the vehicle tracking system. The application has been developed using the google maps APIs. On the Google Maps applications, the objects whose position is detected are called "Markers" or "Points". The shape of this marker can be changed at will. For instance, confusions can occur on a system with a fleet of vehicles, as there are many and various vehicles on the system. In such a case, vehicles can be classified and many different markers can be used depending on the type (Car, shuttle bus, TIR etc.) of the vehicle. This prevents the confusions.

$\mathrm{X}$ and $\mathrm{Y}$ coordinates information comes to the application over GPS. This information is firstly saved in the database created in MYSQL. By virtue of the information saved, the position is marked on the application. With each coordinates saved on the database the application is continuously updated and the tracking of the vehicles is accomplished. This application runs on every computer with internet connection and the vehicles can be tracked regardless of time and location. In Fig. 4 however, display of the web application on a mobile platform is shown. Android has been selected as the mobile platform. The reason for this is that Android operating system is used on 70-80\% of the mobiles devices in the World. The android application of the vehicle tracking system works in the same logic as the web application. The position data coming from the wireless sensor networks are saved in the database. The saved position data can be collected by means of the mobile application and marking and tracking on the map are performed. On the mobile platform as well, the maps of Google have been used. Because of the fact that it is easy to use, is updated rapidly and a giant company like Google always updates these maps, Google maps have been preferred. If desired, different licenced maps can be used.

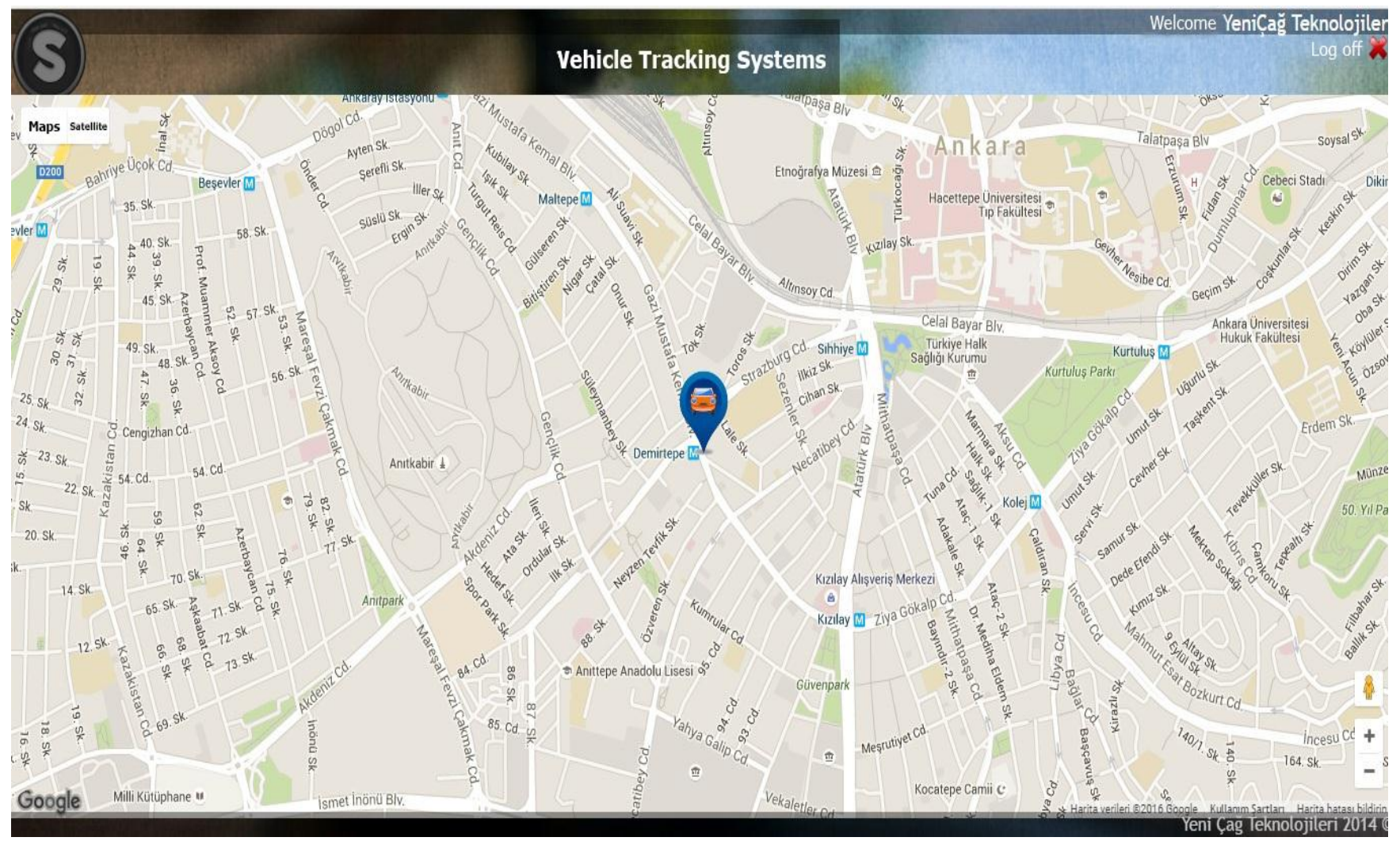

Fig. 3. Vehicle tracking system web application.

With the $\mathrm{X}$ and $\mathrm{Y}$ coordinates information kept on the database instantaneous displaying and tracking can be made as well as daily, weekly, monthly or annual position information can be listed in a graph or a table. Scenario option is available in the features of the Vehicle Tracking System and thanks to this feature, conditioning of the vehicle such as staying in a boundary or not over speeding are also possible. If vehicles speed or get outside the boundaries specified, a message can be sent to the administrator by virtue of the GSM module on the wireless sensor network, call option can be activated, or a mail notification can be initiated.

\section{HuMAN AND OBJECT TRACKING SYSTEM}

The human and object tracking systems are one of the tracking systems of which the use has increased recently. For the safety of children, these systems can be easily used by the parents who would like to trace their children when they go school, private lessons or anywhere. In other words, when a businessman wishes to send his briefcase in which secret information is kept to somewhere else, the tracking of the briefcase and ensuring the safety of the briefcase is also performed by virtue of these systems. The working principles 
of the human and object tracking systems are same as that of the vehicle tracking systems. The only difference between them is that the vehicle tracking systems can be run through the battery of the vehicle. However, in human and object tracking system no such thing is possible. The human and object tracking systems run on the batteries installed in the device. The human and object tracking systems save the data

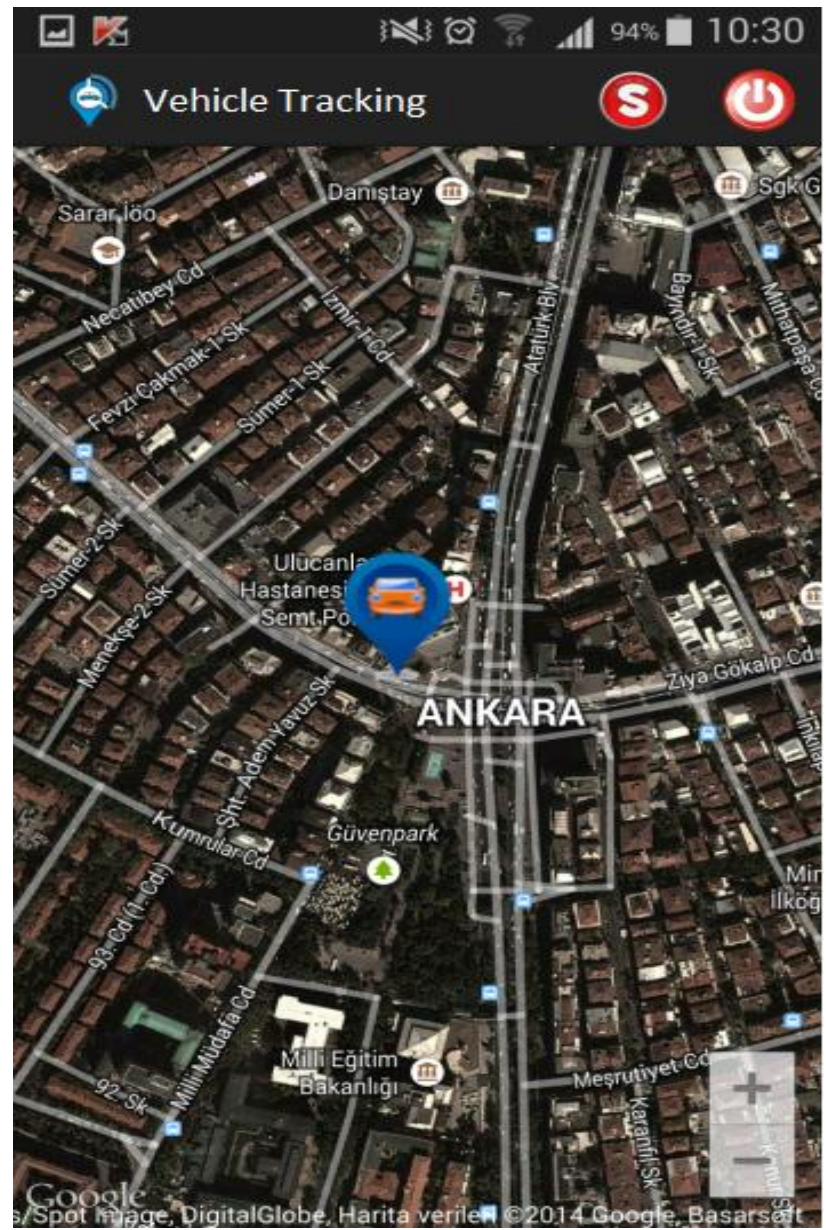

Fig. 4. Vehicle Tracking System Mobile Application. coming from the GPS module as in the vehicle tracking system. With the position information saved in the database, the position is detected on the map with the help of the web application or the mobile application and the tracking is performed. In Fig. 5 and Fig. 6, the applications of the human and object tracking system on web and mobile platforms are shown.

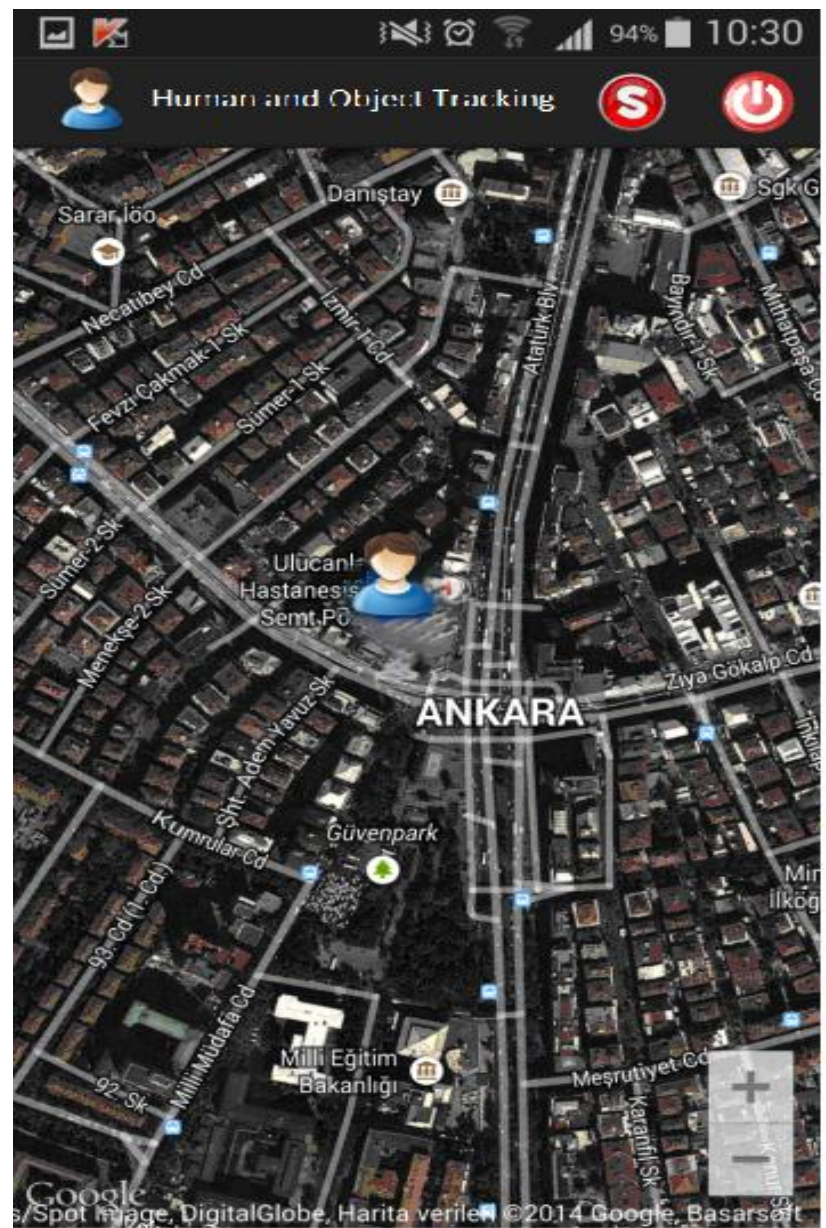

Fig. 6. Human and Object Tracking System Mobile Application.

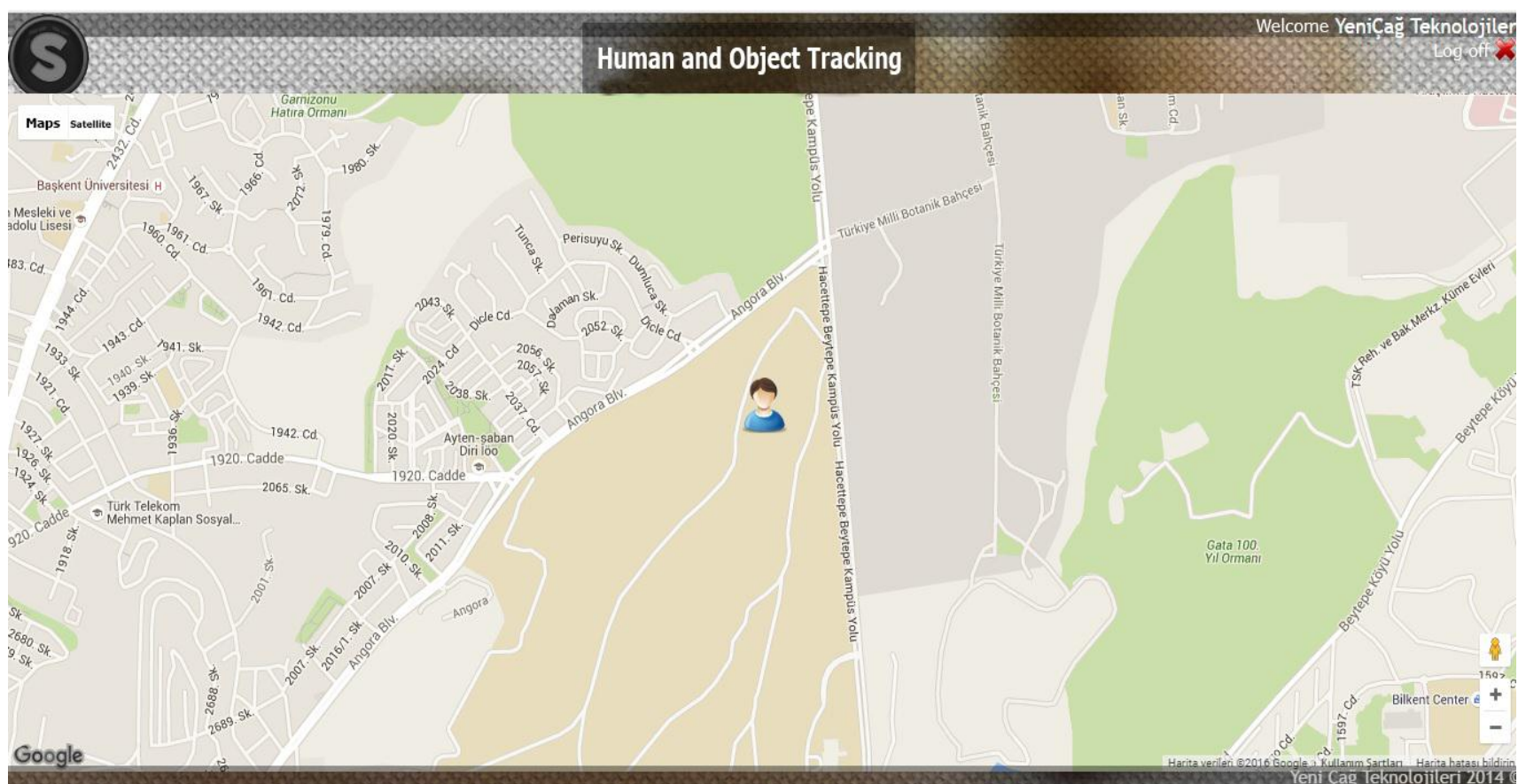

Fig. 5. Human and object tracking system web application 


\section{CONCLUSION}

In this study, displaying of position data collected from the system made up of wireless sensor networks on the web and mobile platforms is implemented. As the icons shown on the map are transiently updated the object on which the tracking device is installed can easily be tracked. As the application gets in contact with the web services, it does not get affected by the changes made in the database. This way, when there is a change in the database section of an application loaded in application store, the application can run without needing for an update.

\section{REFERENCES}

[1] Smartphone OS Market Share, IDC. [Online]. (2015). Available: http://www.idc.com/prodserv/smartphone-os-market-share.jsp

[2] J. F. DiMarzio, Android A Programmers Gulde, McGraw Hill Professional, 2008.

[3] I. F. Akyildiz, W. Su, Y. Sankarasubramaniam, and E. Cayirci, "A survey on sensor networks," IEEE Communications Magazine, vol. 40, no. $8,102-114,2002$.
[4] IEEE-TG15.4, "Part 15.4: Wireless Medium Access Control (MAC) anf Physical Layer (PHY) Specifications for Low-Rate Wireless Personel Area Networks (LR-WPANs)," IEEE Standard for Information Technology, 2003.

[5] A. Koubaa, M. Alves, amd E. Tovar, "IEEE 802.15.4: a Federating Communication Protocol for Time-Sensitive Wireless Sensor Networks," Sensor Networks and Configurations: Fundamentals, Techniques, Platforms and Experiments, Springer-Verlag, Germany, pp. 19-49, 2007.

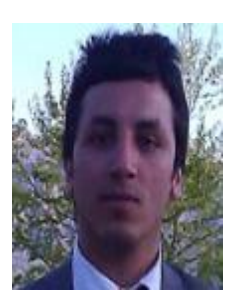

Murat Dener received his M.Sc. and Ph.D. degrees in Electronic and Computer Education Department from Gazi University, Turkey, in 2008 and 2012, respectively. His doctorate thesis entitled by Design and Implementation of a Secure Data Link Layer Protocol for Wireless Sensor Networks. He worked in Georgia Tech E-Stadium Team in 2011. From 2005 to 2012, he was a Research Assistant in the Graduate School of Natural and Applied Sciences. Since 2012, he is Doctor in Gazi University. His research interest includes the next-generation wireless networks, wireless ad hoc and sensor networks, cognitive radio networks. 